\title{
Review paper on Software Data Reduction for Bug Triage
}

\author{
Miss. Karande Rupali .D ${ }^{1}$, Mr. H.P. Khandagale ${ }^{2}$ \\ Student, Computer Science and Technology, Department of Technology (SUK), Kolhapur, India ${ }^{1}$ \\ Asst. Prof, Computer Science and Technology, Department of Technology, Kolhapur, India ${ }^{2}$
}

\begin{abstract}
Bug is a very essential factor which is occur in software. Almost every company facing problem to solve issues related bug, software companies spends over 40-45 percent of cost in dealing with software bugs. Very important step to handle bug is bug triage, which aims to correctly assign a developer to a new bug. To lower the time price in guide work, text category strategies are implemented to behavior computerized bug triage. In this paper, we address the problem of facts reduction for bug triage, i.e., a way to reduce the scale and enhance the satisfactory of bug records. We integrate instance selection with feature selection to simultaneously lessen facts scale on the malicious program dimension and the word measurement. To determine the order of making use of example selection and function choice, we extract attributes from historic malicious program information units and build a predictive model for a new computer virus information set.
\end{abstract}

Key words: Mining software repositories, application of data preprocessing, data management in bug repositories, bug data reduction, feature selection, instance selection, bug triage, prediction for reduction orders.

\section{INTRODUCTION}

For handling these software program application repositories, the computer virus repositories play a very critical feature in extraction of the information. Computer virus repositories consist of all bug reports and these computer virus repots are mapped as a file and a particular developer is mapped to the label of the document. Developers similarly to users can put up their defects thru massive open supply task like Mozilla and Firefox due to the truth they comprise large laptop virus repositories to keep all of the computer virus reports. The normal happening error are so huge that it turns into too tough to address the specific issue. The principle objective of the paper is to gain the bug reviews from large informantion units. To get the correct outcomes we're going to get low scale and high exceptional data units with the aid of doing away with the computer virus reviews and phrases which can be redundant and so as to be non-informative. By way of using this method we're going to boom the accuracy of the computer virus reports. We are also set to offer the worm reviews according to a particular area, that's no matter the domain which a enterprise o person may be using for his mission we're going to tackle the results consistent with that specific domain. Bug reports are produced in step with that domain using pinnacle-k pruning set of rules which tackles each report with the help of a rating system.

Bug repository (a standard software program repository, for storing information of insects), plays crucial role in dealing with software program bugs. Software bugs are inevitable and fixing insects is highly-priced in software program improvement. Software groups spend over 45 percentage of value in fixing bugs. massive software initiatives installation bug repositories (also referred to as bug tracking systems) to help facts collection and to help builders to deal with bugs .In a worm repository, a computer virus is maintained as a bug file, which facts the textual description of reproducing the bug and updates according to the repute of bug fixing. A bug repository affords a statistics platform to support many styles of tasks on insects, e.g., fault prediction computer virus localization, and reopened worm analysis. In this paper, malicious program reviews in a worm repository are called computer virus information. There are two demanding situations related to computer virus facts that may affect the powerful use of computer virus repositories in software program development responsibilities, specifically the huge scale and the low great. On one hand, because of the everyday-suggested insects, a large quantity of latest bugs are stored in bug repositories.

Bugs might also deceive related builders at the same time as redundant insects waste the restrained time of malicious program handling. A time-ingesting step of handling software program insects is worm triage, which objectives to assign an accurate developer to restoration a brand new worm. In conventional software improvement, new bugs are manually triaged via an expert developer, i.e., a human cause. Due to the large wide variety of every day insects and the lack of awareness of all the insects, manual malicious program triage is steeply-priced in time value and occasional in accuracy. In guide bug triage in Eclipse, forty four percent of insects are assigned by using mistake at the same time as the time price between starting one computer virus and its first triaging is 93 days on common. To keep away from the pricey cost of guide computer 
virus triage, current paintings has proposed an automated 5. Memories of bug fixes

bug triage approach, which applies textual content In this paper, specialists discover the bug locating device category strategies to expect builders for bug reviews. On to find the quantity of occurrences the unique bug this technique, a worm file is mapped to a file and document has arrived. Research display that $35 \%$ of the associated developer is mapped to the label of the bugs occur again and again. To store the history of the document. Then, malicious program triage is transformed incidence of these types of computer virus reviews and to right into a trouble of text type and is mechanically solved hold a backup copy, we use a source code repository. with mature textual content type techniques, e.g., Naive Through using the stored statistics, this paper offers the Bayes. Based totally at the consequences of text type, a complete expertise approximately the records of the human triager assigns new bugs by means of incorporating appearance ofinsects to the developers.

his/her knowledge

\section{LITERATURE REVIEW}

Within the current situation, experts have been the folks who used to robotically assign a developer to restore a bug. This phase presents the associated paintings executed, which indicates the utilization of various techniques that were executed to boom the accuracy of the worm reviews.

\section{Automatic bug triage using text categorization.}

On this paintings, the specialists used the textual content type technique that's the Naive Bayers method that's used to classify the text based totally on their information units. However as they've now not used some other method the ideologies in this paper have did not attain the most accuracy that have to had been met. Best $25 \%$ of the accuracy is been capable of meet inside the above stated paper.

2. A Framework for automatic assignment of bugs using vector space method.

Vector area model is a model which incorporates the history or the expectances of all of the builders which might be been able to fix a malicious program. On this version which uses the vector method, the histories of the builders are fetched and the bugs were robotically assigned. However this approach also didn't meet the accuracy level.

\section{Improving bug triage using bug tossing graphs.}

In this paper the specialists have surveyed over four, 45,678 bug reports from huge open source projects. Studies shows that this technique consumes plenty of time as it makes use of the tossing version that's used to assign the best developer. Time consumption is the predominant disadvantage and additionally it fails to satisfy the accuracy degree as its miles of using greater strategies to improve the bug reports.

4. A cost aware bug triage algorithm for bug reporting In this paper the experts have used a value triaging approach. The principle drawback of this paper is that fee is effectively reduced by accuracy is once more a query of fact. Here the whole cost that is taken to fix a specific computer virus is taken under consideration. Because the accuracy of the computer virus reports isn't yet met, we cannot take the ideology that is been depicted on this paper.

\section{Bug triage with software data reduction techniques} The above paper offers the whole records to boom the accuracy of the worm reports. Here the usage of example Selection and characteristic choice set of rules is been cited and the order of applying these set of rules is likewise given. We additionally prepare a predictive version which routinely predicts the order of making use of the algorithm.

\section{PROPOSED SYSTEM}

Within the information discount manner, the principle aim is to reduce to track bug and send to developer. When bug is track, it will send to developer for removing it from program. After this process, if again error occurred during program, we will proposed method in which error will be automatically removed from program.

The purpose why we use each example choice and function choice set of rules is that if we use most effective example choice then we can decrease the worm reports however accuracy is reduced. If we use handiest the characteristic choice then we will get accelerated accuracy however phrases are decreased. Combination of both instance and function choice we lessen the computer virus reports and words on the equal time growth the accuracy.

\section{BASIC SYSTEM ARCHITECTURE}

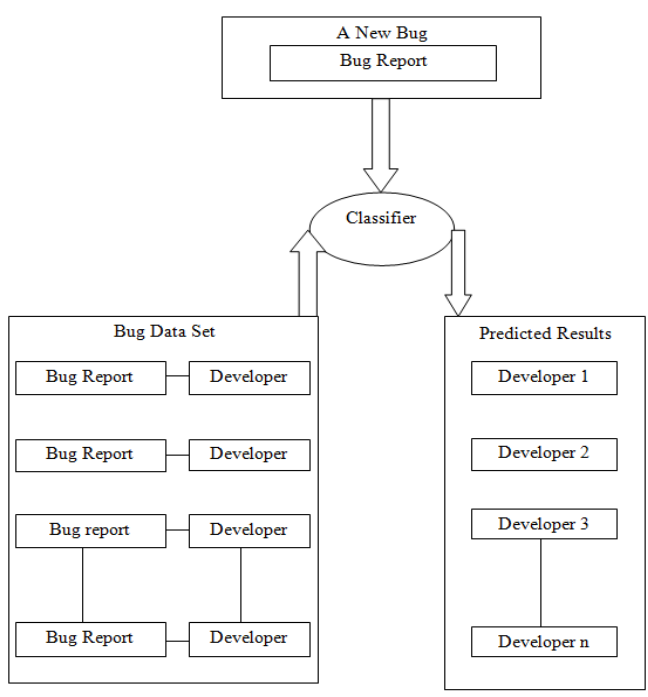

Fig1: Architecture of Proposed System 


\section{MODULES}

- Instance selection:

Instance choice and characteristic selection are widely used techniques in statistics processing. For a given information set in a sure software, instance choice is to obtain a subset of applicable times (i.e., worm reviews in bug facts) while feature selection objectives to attain a subset of relevant capabilities (i.e., words in bug facts). In our paintings, we rent the mixture of instance choice and feature selection.

- Statistics reduction:

In our paintings, to save the hard work cost of developers, the records reduction for bug triage has dreams.

1) decreasing the statistics scale.

2) enhancing the accuracy of malicious program triage.

In comparison to modelling the textual content of bug reports in current work, we intention to augment the data set to build a preprocessing approach, which can be implemented earlier than an existing bug triage technique. If bug will be automatically removed through system from program.

\section{CONCLUSION}

Computer bug triage is an pricey step of software program upkeep in each labor price and time cost. on this paper, we combine function choice with example selection to lessen the dimensions of worm information sets in addition to enhance the facts high-quality. To decide the order of applying example selection and function selection for a new computer virus records set, we extract attributes of every worm facts set and teach a predictive version based totally on ancient facts sets. We empirically look at the information reduction for trojan horse triage in trojan horse repositories of two big open supply tasks, particularly Eclipse and Mozilla. Our work offers an method to leveraging strategies on facts processing to form reduced and awesome trojan horse statistics in software improvement and preservation. In future paintings, we plan on enhancing the effects of information reduction in computer virus triage to explore the way to prepare a high satisfactory bug records set and tackle a website-precise software program task. For predicting reduction orders, we plan to pay efforts to discover the capacity dating between the attributes of bug records units and the discount orders.

\section{REFERENCES}

[1] J. Anvik, L. Hiew, and G. C. Murphy, "Who should fix this bug?" in Proc. 28th Int. Conf. Softw. Eng., May 2006, pp. 361-370.

[2] S. Artzi, A. Kie_zun, J. Dolby, F. Tip, D. Dig, A. Paradkar, and M. D. Ernst, "Finding bugs in web applications using dynamic test generatio and explicit-state model checking," IEEE Softw., vol. 36, no. 4, pp. 474-494, Jul./Aug. 2010.

[3] J. Anvik and G. C. Murphy, "Reducing the effort of bug report triage: Recommenders for development-oriented decisions," ACM Trans. Soft. Eng. Methodol., vol. 20, no. 3, article 10, Aug. 2011.
[4] C. C. Aggarwal and P. Zhao, "Towards graphical models for text processing,” Knowl. Inform. Syst., vol. 36, no. 1, pp. 1-21, 2013.

[5] Bugzilla, (2014). [Online]. Avaialble: http://bugzilla.org/

[6] K. Balog, L. Azzopardi, and M. de Rijke, "Formal models for expert finding in enterprise corpora," in Proc. 29th Annu. Int. ACM SIGIR Conf. Res. Develop. Inform. Retrieval, Aug. 2006, pp. 43-50.

[7] P. S. Bishnu and V. Bhattacherjee, "Software fault prediction using quad tree-based k-means clustering algorithm," IEEE Trans. Knowl. Data Eng., vol. 24, no. 6, pp. 1146-1150, Jun. 2012.

[8] H. Brighton and C. Mellish, "Advances in instance selection for instance-based learning algorithms," Data Mining Knowl. Discovery, vol. 6, no. 2, pp. 153-172, Apr. 2002.

[9] S. Breu, R. Premraj, J. Sillito, and T. Zimmermann, "Information needs in bug reports: Improving cooperation between developers and users," in Proc. ACM Conf. Comput. Supported Cooperative Work, Feb. 2010, pp. 301-310.

[10] V. Bol_on-Canedo, N. S_anchez-Maro no, and A. AlonsoBetanzos, "A review of feature selection methods on synthetic data,” Knowl. Inform. Syst., vol. 34, no. 3, pp. 483-519, 2013.

[11] V. Cerver_on and F. J. Ferri, "Another move toward the minimum consistent subset: A tabu search approach to the condensed nearest neighbor rule," IEEE Trans. Syst., Man, Cybern., Part B, Cybern.,vol. 31, no. 3, pp. 408-413, Jun. 2001.

[12] D. Cubrani_c and G. C. Murphy, "Automatic bug triage using text categorization," in Proc. 16th Int. Conf. Softw. Eng. Knowl. Eng.,Jun. 2004, pp. 92-97.

[13] Eclipse. (2014). [Online]. Available: http://eclipse.org/

[14] B. Fitzgerald, "The transformation of open source software," MIS Quart., vol. 30, no. 3, pp. 587-598, Sep. 2006. 